\title{
Crowdsourcing platform: a review for governing operating model
}

\begin{abstract}
In recent years, the emergence of crowdsourcing platform as a mediator in managing the intersection of business organization and human workforce via the Internet has paved way for business process to spur globally as it requires minimal cost expenditure and faster. However, due to its vast concept and applied in various directions and fields in research, it has led to an obscure limit. Hence, many believe that there are many opportunities still remain undiscovered. This paper aims to gain a better understanding of the governing operating model in crowdsourcing from the perspective of Information Communication and Technology and business process. We conducted a review and analyses of previous studies related to crowdsourcing platforms. Specifically, this paper deliberates on the main components in such model then analyzed the intersection between each component in order to facilitate dynamic changes in crowdsourcing operating platform. With such discovery, the governing operating model of crowdsourcing platform is hopefully can be further enhanced in the near future.
\end{abstract}

Keyword: Crowd-sourced process; Crowdsourcing platform; Tasks structure 